\title{
Estrategias no convencionales de servicio: Un desafío en el diseño organizacional
}

\author{
Francisco Vergara Streinesberger ${ }^{1}$ \\ Juan Carlos Robledo Fernandez ${ }^{2}$ \\ Zully Aguilera Prins ${ }^{3}$ \\ Gustavo Camargo Gutierrez ${ }^{4}$
}

\section{Resumen}

Las empresas existen para crear valor y viven para crear riqueza, sin embargo estos propósitos hoy resultan cada vez más complejos de lograr de manera óptima, en virtud de las dificultades, rivalidad y condiciones limitadas donde operan todas las organizaciones en un mundo cada vez más globalizado. Las personas siguen siendo un fin y un medio para las empresas, en este contexto, el rol de clientes que estos desarrollan, es determinante del margen que obtienen las empresas que producen y prestan servicios para satisfacerlos. Este proceso se encuentra mediado por los gustos, preferencias, cultura, valores, principios y demás elementos que constituyen el perfil de los consumidores reales y potenciales, por lo tanto las empresas han reconocido la importancia de crecimiento hacia fuera, fundamentado en una efectividad productiva que los respalde, una logística externa, marketing, ventas y en especial un servicio diseñado y desarrollado con enfoque diferenciado para un cliente emocionalmente diferente y que posee un esquema complejo de necesidades, coherentes en términos básicos, pero particulares a nivel superior.

\section{Palabras Claves}

Estrategias no convencionales de Servicio, modelo teórico, servicio, estrategia, conexión emocional, diseño organizacional.

\begin{abstract}
Organizations exist for the purpose of creating value and live for creating richness. Nevertheless these purposes today result more and more complex to achieve optimally, due to difficulties, rivalries and limited conditions where all organizations work in a more globalized world. People are still an end and a means for the enterprises; in this context the customer roll they develop is determining for the revenue of this enterprises which produce and provide services to satisfy them. This process is determined by tastes, preferences, culture, values, and principles among other elements that constitute the real and potential customers profile, therefore the companies have recognized the importance of the inside-out growth, founded on a backed up productive effectivity, external logistics, marketing, sales and above all a special service designed and developed on a differentiated approach for a customer emotionally different who possess as well a complex scheme of needs, coherent in a basic level but particular in a higher one.
\end{abstract}

\section{Keywords}

Non-conventional service strategies, theoretical service model, service, strategy, emotional connection, organizational design.

Fecha de recepción: 11 de Diciembre de 2018, Fecha de evaluación: 7 de Enero de 2019, Fecha de aceptación: 4 Marzo de 2019

\footnotetext{
1. Magister en administración. Ingeniero mecánico. Correo electrónico: francisco.vergara@estreico.com

2. Phd. en administración. Magister en administración. Economista. Vinculado a la Corporación Universitaria Americana.

Correo electrónico: jrobledo1962@gmail.com

3. Magister en administración. Ingeniera industrial.

4. Magister en administración. Correo electrónico: gustavocamargogutierrez@gmail.com
} 
Francisco Vergara Streinesberger, Juan Carlos Robledo Fernandez, Zully Aguilera Prins, Gustavo Camargo Gutierrez

\section{Introducción}

El sector de los servicios en el marco global, ha venido evolucionando lentamente desde el fin de la revolución industrial, llegando a ocupar el primer lugar en las economías globales. Tanto así que en la actualidad, en Estados Unidos el sector de servicios representa el $80 \%$ de su PNB (Frei \& Morris, 2012). Este fenómeno ha ocasionado que los diferentes mercados han sido conducidos hacia un entorno altamente competido, y en consecuencia caracterizados por el constante incremento en las expectativas de los clientes, lo que hace que las organizaciones se vean en la necesidad de adoptar estrategias cada vez más agresivas e innovadoras, que permitan reducir la brecha entre los servicios percibidos y esperados por los usuarios (Parasuraman et al, 1985).

Sin embargo, proporcionar un servicio de alta calidad implica frecuentemente incurrir en costos de operación elevados, lo cual crea una paradoja económica en la oferta de servicio, pues la satisfacción del cliente es uno de los pilares que enmarcan la rentabilidad (Hallowell, 1985) y por lo tanto la sostenibilidad de las empresas de servicio, por tanto la satisfacción tiene un costo alto y muchas empresas han fracasado financieramente buscando la perfección en el servicio siguiendo el tradicional precepto de “el cliente siempre tiene la razón”(Albrecht \& Zemke, 2000). Esto hace que en el mundo moderno, las estrategias de servicio deban estar fundamentadas desde un punto de vista holístico, teniendo en cuenta aspectos claves como los recursos y capacidades, la calidad del servicio y el desempeño (Mukherjeel, P Nath and M Pal, 2003), además de los necesarios aspectos financieros.

En las organizaciones que desarrollan estrategias de servicios, un aspecto esencial para la gestión es la medición de la calidad del ser-

vicio que se está proporcionando, para lo cual tradicionalmente se siguen dos corrientes teóricas: el modelo SERVQUAL (Parasuraman et al, 1985) y el modelo SERVPERF (Cronin y Taylor, 1992), los cuales proponen una medición de la calidad del servicio y el desempeño de los procesos; estos modelos son una aproximación subjetiva basada en expectativas y apreciaciones de los consumidores, aunque no tienen en cuenta los aspectos financieros ni emocionales de la operación. Desde el punto de vista financiero de la calidad del servicio, (Rust et al, 1995) proponen una forma de medir el retorno sobre la calidad (ROQ), lo cual permite una mejor aproximación al objetivo financiero de las organizaciones. De igual forma, Fleming et al. (2005) establecen una relación lineal entre la actitud de los empleados, los requerimientos de los clientes y el desempeño financiero.

Con base en los elementos anteriores, el problema que se plantea en la presente investigación es que las organizaciones afrontan un dilema permanente en términos de brindar un excelente servicio, pero a su vez mantener sus costos de operación en niveles que permitan la sostenibilidad y rentabilidad de la operación. Esto obliga a las empresas a buscar estrategias innovadoras que se aparten de los métodos tradicionales con el fin de lograr un factor diferenciador eficiente en términos de resultados financieros y a la vez de satisfacción de los clientes. Para resolver este problema se requiere observar algunos casos de éxito o fracaso en empresas globales que permitan evidenciar las mejores prácticas que se utilizan en la actualidad y cómo estas han sido determinantes en sus resultados financieros y de esta manera plantear la aproximación a un modelo teórico que pueda agrupar estas tendencias. 


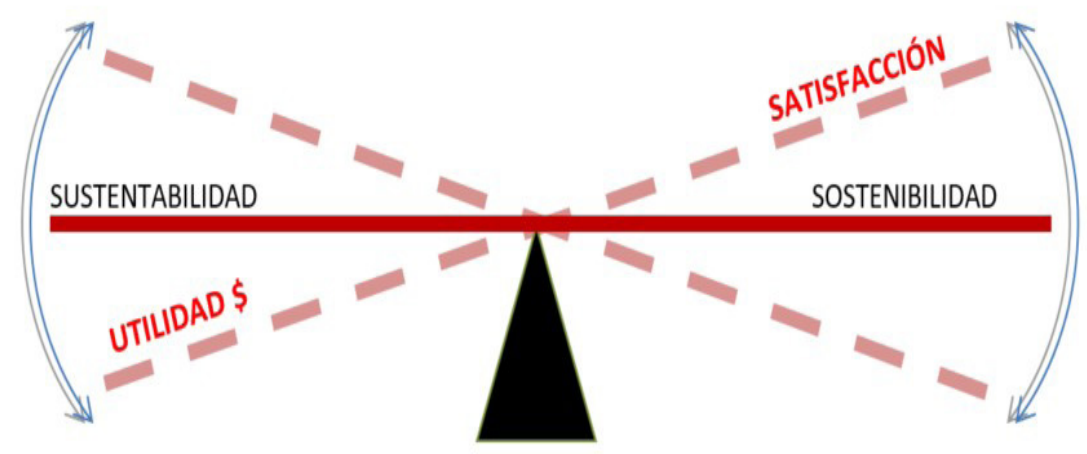

Figura1. El dilema entre satisfacción y utilidad.

Fuente: Elaboración propia

\section{Fundamento teórico}

Estrategia es un término muy usado en el mundo empresarial y organizacional, hoy en día es muy común que las grandes iniciativas entrañen en su esencia estrategias (De Ríos, 2018); desde la gestión de proveedores, la cual da soporte vital para todos los procesos de la organización (Sarmiento, 2018), hasta las estrategias orientadas a la calidad máxima en el servicio. Respecto a esta última se comienza a hablar de ello a finales de la década de los 70 (Passer, Olsen y Wickoff 1978, Gronross 1982, Lehtinen y Lehtinen 1982, Lewis y Booms 1983) cuando el servicio empieza a tomar importancia en el mercado global como valor agregado de las organizaciones, trascendiendo a la misma calidad de los productos ofrecidos, pues hasta ese entonces, la literatura hablaba independientemente de calidad, de estrategia o de servicio, pero como temas no relacionados.

De otra parte, la literatura relacionada con la calidad se había enfocado históricamente desde un punto de vista de los productos, con las teorías clásicas de Deming (1950) que dieron inicio a la cultura mundial de la calidad. Si bien se encuentra en la bibliografía algunos escritos alrededor de la descripción de los servicios ya desde finales de la década de los 70's (Baterson 1977, Shostak 1977, Berry 1980, Lovelock 1981), Uno de los primeros escritos modernos relacionados con modelos de gestión de la calidad del servicio lo plantearon Parasuraman, Zeithaml y Berry (1985) a través del modelo SERVQUAL y Cronin y Taylor (1992) con el modelo SERVPERF. Más reciente es el modelo del triángulo del servicio planteado por Albrecht y Zemke (2003).

Para realizar una primera aproximación a la calidad del servicio, es necesario entender tres de sus conceptos básicos: las características de intangible, heterogéneo e inseparable (Parasuraman, Zei-thaml, \& Berry 1985). La primera característica del servicio es que es intangible, es decir que no se puede tocar ni almacenar. Se produce justo en el momento en el que se entrega y normalmente es producido y entregado por seres humanos (salvo excepciones como un cajero automático), lo cual conlleva a la segunda característica de heterogeneidad, es decir que el servicio producido y entregado puede variar de un día a otro, de una persona a otra, de un cliente a otro pues aunque se hayan trazado métodos y estándares de producción del servicio, este es inherente al ser humano y por consiguiente está sujeto a sus propias emociones, las cuales pueden variar dependiendo del día, hora, condiciones, interacciones, etc. Finalmente, el servicio es inseparable de su fuente; esto quiere decir que se produce en el mismo instante en que se consume, por lo tanto no se pueden fabricar en masa o en ninguna línea de producción, aun cuando se pueda producir a través de máquinas, como en el ejemplo anterior de un cajero automático, una máquina de pago automático, una página de Internet, etc., lo cual refuerza su primera característica de intangibilidad. 
Francisco Vergara Streinesberger, Juan Carlos Robledo Fernandez, Zully Aguilera Prins, Gustavo Camargo Gutierrez

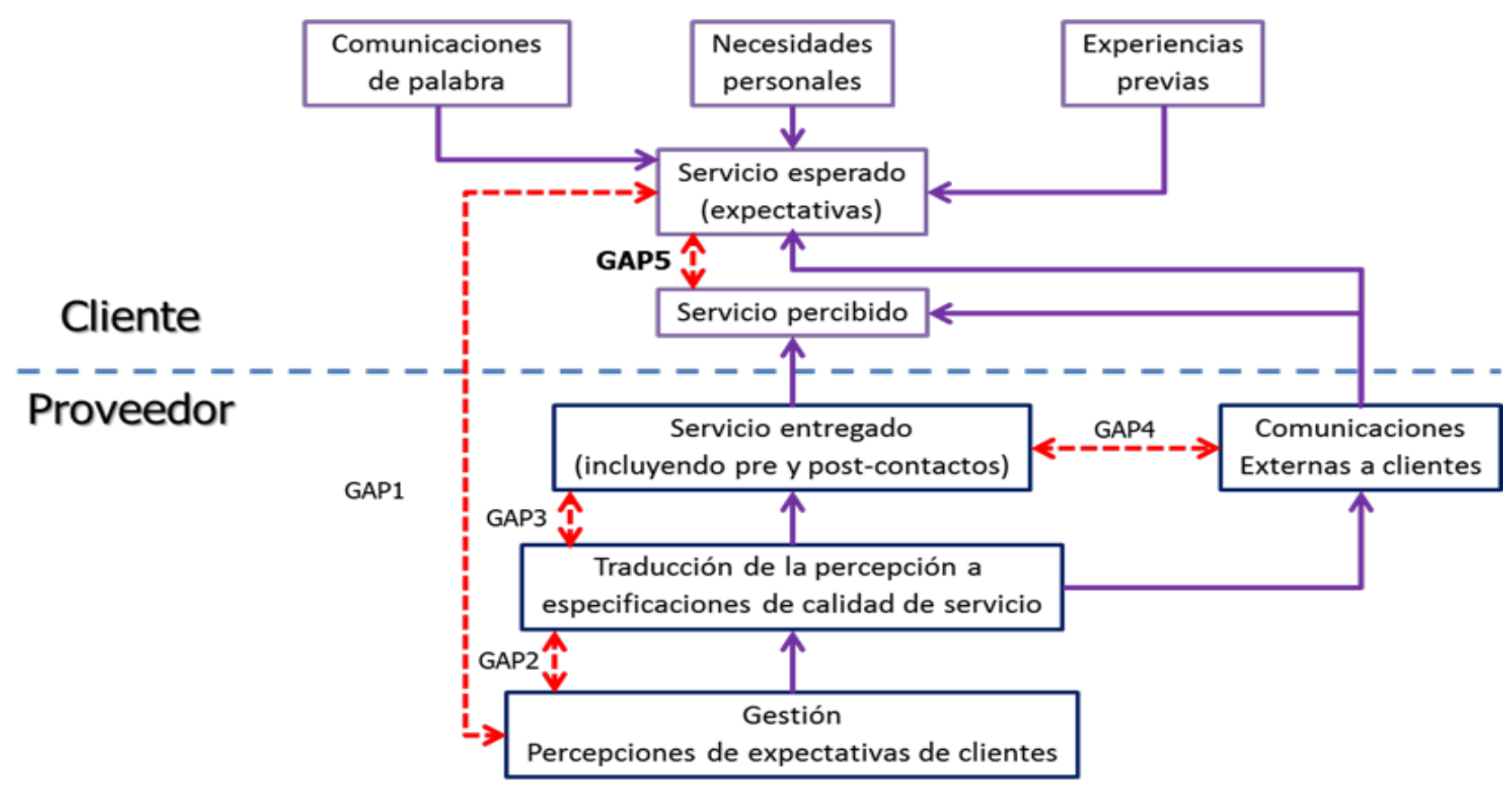

Figura 2. Modelo SERVQUAL.

Fuente: A. Parasunaman, 1985 (traducción del autor)

Bajo las premisas de este modelo, el servicio se puede calificar como bueno cuando el servicio entregado cumple con las expectativas del usuario. Además se puede lograr el calificativo de excelente, cuando el servicio entregado logra exceder las expectativas del usuario. Por ejemplo en un servicio de reparación técnica, el técnico no solamente puede arreglar el problema del aparato, sino ir más allá y explicarle al cliente las razones por las que pudo haber ocurrido un fallo y qué puede hacer el usuario para evitar futuros daños o para corregirlos el mismo. Sin embargo esto depende mucho de la disposición del personal de servicio a "ir más allá", lo cual hace del servicio una actividad difícil de ejecutar de acuerdo a los estándares definidos por una organización.

Por su parte, el modelo SERVPERF de Cronin y Taylor (1992) se enfoca hacia la medición de la calidad del servicio basada en el desempeño y en el cual se argumenta que la medida de la diferencia entre desempeño y expectativas es una base inapropiada en la medición de la calidad del servicio, en contraposición al modelo SERVQUAL. $\mathrm{Su}$ estudio sugiere que la calidad del servicio debe ser medida como una actitud, puesto que si bien la calidad del servicio es un antecedente para la satisfacción del cliente y que esta a su vez ejerce una influencia más fuerte en las intenciones de compra que la misma calidad del servicio.
Complementariamente existen otros factores que influyen en las intenciones de compra como la conveniencia, precio o disponibilidad, factores que son independientes de la calidad del servicio.

Paralelamente, Bolton y Drew (1991) definen dos conceptos separados: Satisfacción y Actitud. La Satisfacción se refiere a la evaluación del cliente basada en su experiencia posterior al servicio, mientras que la Actitud se refiere a la evaluación global de la oferta del servicio, incluyendo las expectativas previas a la ejecución. También definen la percepción residual como la imagen mental de la evaluación del cliente hacia la oferta de servicio, que puede permanecer en su memoria durante algunos meses, afectando las actitudes futuras hacia el mismo servicio. En su investigación comprobaron la existencia de un vínculo entre la calidad del servicio, en términos de atributos específicos, y la actitud del cliente hacia dicho servicio y que dicha actitud es una función tanto de la percepción residual como de su nivel de satisfacción o insatisfacción por el desempeño actual del servicio, de tal forma que un cambio en los niveles o atributos del servicio tendrán un efecto de más largo plazo en el impacto sobre la satisfacción de los clientes, debido a que también se encuentra fuertemente influenciada por el efecto residual de experiencias previas, ya sean positivas o negativas, que proporciona un efecto 
de corto plazo en las actitudes de los usuarios en experiencias futuras. Por lo tanto en el diseño de una nueva estrategia de servicio o en el ajuste de una ya existente deberá considerarse que su impacto sobre las actitudes de los clientes se logrará solamente en el mediano o largo plazo, debido al efecto residual de experiencias previas en la percepción de los clientes.

Lovelock et. al (2004) plantean que los servicios incluyen a menudo elementos tangibles, como la cama del hotel, la máquina del gimnasio, el repuesto utilizado para reparar un equipo, etc. Sin embargo es el desempeño del servicio lo que le da su principal característica de intangible. Por su parte, Kottler (1997) define cinco categorías para diferenciar un producto de un servicio el cual puede ser un elemento mayor o menor dentro de la oferta total:

- Bien puramente tangible (como jabón, sal, crema dental), en el cual ningún servicio acompaña al producto.

- Bien tangible con servicios que lo acompañan (por ejemplo automóviles o computadoras). En estos, los servicios asociados (entrega, reparaciones, mantenimiento, capacitación, garantía, etc.) ayudan a hacer más atractivo el producto.

- Híbrido (por ejemplo un restaurante), que combina partes iguales de bienes y servicios y el impacto de ambos es igual de importante en la experiencia y valor entregado al cliente.

- Servicio fundamental con bienes y servicios menores que lo acompañan (por ejemplo un viaje aéreo, en la que el bien principal es un avión pero el beneficio fundamental es un servicio, y también incluye bienes y servicios de apoyo como la comida a bordo, revistas, etc.)

- Servicio puro (por ejemplo el cuidado de niños, masaje o la psicoterapia). En estos no existe ningún producto asociado, más allá del espacio físico donde se ejecuta el servicio.

Existen también servicios con mucho o poco contacto, tanto del personal de servicio con el cliente como entre clientes, por ejemplo en un autobús o un estadio deportivo en donde la experiencia del cliente depende a su vez del comportamiento de los demás clientes. En la medida en que el contacto personal sea más frecuente, el objetivo de llegar a la satisfacción se vuelve cada vez más retador y complejo. (Lovelock et al., 2004).

En cuanto a las conexiones emocionales, los seres humanos, desde la perspectiva socioeconómica, tienen una razón de ser caracterizada por los intereses de satisfacer sus necesidades. Por lo regular de manera individual es imposible hacerlo y por lo tanto se organizan y relacionan con empresas, para obtener las condiciones y los bienes o servicios que utilizan como instrumento, para satisfacer sus necesidades. Sin embargo es importante aclarar que algunas necesidades van más allá de lo tangible y como tal son los servicios los medios. Entonces resulta importante entender las necesidades, su clasificación y en particular como esta información es clave para el diseño del servicio, con fines de comercialización, cuyos resultados, que son la creación de valor, las ventas y la satisfacción del comparador, se encuentran mediados por conexiones emocionales que se construyen en la mente del consumidor con respecto al bien o al servicio.

Hablar de necesidades, implica traer a colación el marco conceptual propuesto por Maslow (1954) en sus diferentes trabajos, en especial la jerarquización que realizo de estas. Este en sus estudios con monos en laboratorios norte americanos, se percató que existen necesidades más importantes que otras, lo cual implica la prioridad de ser satisfecho y como tal el orden estratégico de actividades para lograrlo. En otros casos el estableció que el contexto, el entorno y las condiciones del individuo que intenta satisfacer sus necesidades, también resulta influir en la importancia clasificación que este realice con respecto a ellas.

Boeree (2006), afirma que Maslow recogió esta idea y creó su ahora famosa "jerarquía de necesidades". Además de considerar las evidentes agua, aire, comida y sexo, el autor amplió 5 grandes bloques: las necesidades fisiológicas, necesidades de seguridad y reaseguramiento, la necesidad de amor y pertenencia, necesidad de estima y la 
necesidad de autorrealización el sí mismo; en este orden. Logrando compartir y socializar sus teorías en diferentes sus obras, entre las cuales se destacan: Toward a Psychology of Being (1968), Motivation and Personality (firstedition, 1954, and secondedition, 1970), and TheFurtherReaches of Human Nature (1971) Finalmente, hay muchos artículos escritos por Maslow, especialmente en el Journal of HumanisticPsychology, de la que fue co-fundador.

Según este autor, las necesidades del ser humano están jerarquizadas y escalonadas de forma tal que cuando quedan cubiertas las necesidades de un orden es cuando se empiezan a sentir las necesidades del orden superior. Es precisamente estos postulados un punto de partida conceptual clave para el desarrollo de este estudio, en el cual pretendemos formular una hipótesis que conceptualmente categoriza las necesidades en básicas y superiores, pero que al mismo tiempo cree, que el mundo globalizado de hoy, tiene como protagonistas empresas preocupadas más por atender las necesidades superiores, para lo cual resulta clave comprender las emociones y las conexiones emocionales que estratégicamente empresas de talla global, estudian, con la intención de construir ventajas competitivas que se reflejen en su creación de valor por lo tanto en lo sustentable, sostenible y la creación de riqueza de la dicha organización, a partir de la optimización del diseño y del servicio que se preste, luego de conocer emocionalmente a sus consumidores.

El escalón básico de Maslow (1956), es el de las necesidades fisiológicas, hambre y sed. Cuando el ser humano tiene ya cubiertas estas necesidades empieza a preocuparse por la seguridad de que las va a seguir teniendo cubiertas en el futuro y por la seguridad frente a cualquier daño. Confirma Boeree (2006), que una vez que el individuo se siente físicamente seguro, empieza a buscar la aceptación social; quiere identificarse y compartir las aficiones de un grupo social y quiere que este grupo lo acepte como miembro. En este sentido surge la pregunta: ¿qué empresas atienden estas necesidades y que importancia tienen las emociones a la hora de diseñar los bienes o los servicios con que logran o intentan satisfacer dichas necesidades? Estos cuestionamientos son un poco lo que permite en esta investigación reflexionar, sobre la forma en que empresas globales resuelven esta situación.

Cuando el individuo está integrado en grupos sociales empieza a sentir la necesidad de obtener prestigio, éxito, alabanza de los demás. Finalmente, los individuos que tienen cubiertos todos estos escalones, llegan a la culminación y desean sentir que están dando de sí todo lo que pueden. En este contexto se encuentra de manera transversal presente una relación entre las necesidades superiores y las emociones, tomando en cuenta que estas últimas a nivel Conductual, sirven para establecer nuestra posición con respecto a nuestro entorno, y nos impulsan hacia ciertas personas, objetos, acciones, ideas y nos alejan de otros. Las emociones actúan también como depósito de influencias innatas y aprendidas, y poseen ciertas características invariables y otras que muestran cierta variación entre individuos, grupos y culturas (Levenson, 1994).

Si se trata de analizar cómo funciona esta relación, entre las necesidades, las emociones y los servicios o bienes que las empresas generan, se logró identificar lo que se ha entendido como una conexión emocional, que en la medida que las compañías las estudien, entiendan, se informen de ellas y logren construir e implementar estrategias no convencionales que las optimicen, estarán tejiendo factores críticos de éxito o simplemente ventajas competitivas que crean valor. Por ejemplo se pueden encontrar empresas de talla mundial, reconocidas así por la revista "Forbes", que permiten comprender a nivel empírico lo planteado: La Coca-Cola no es un producto para satisfacer la sed sino para satisfacer la necesidad de pertenecer al grupo de jóvenes que se divierten. El Mercedes Benz trata de satisfacer la necesidad de éxito y prestigio social. Apple no produce computadores. Distribuye tecnología, presta servicios informáticos, simplemente vende prestigio; comprende que genera estatus como una condición social que facilita entrar y mantenerse en cierto círculo de la sociedad (necesidad de afiliación).

Las necesidades están asociadas a las carencias que el hombre percibe, y como tal han sido clasificadas desde diferentes perspectivas. En este sentido Maslow habla de las básicas y superiores, 
Herzberg (2009) establece dos tipos de necesidades, las de orden inferior y las superiores, concentrando sus trabajos en los ámbitos laborales $\mathrm{y}$ en especial en el marco de las relaciones empleado - empleador. otras perspectivas categorizan las necesidades en esenciales y ocasionales, complementarias, Primarias y secundarias (naturales y sociales), presentes y futuras, ocasionales e intermitentes, elásticas e inelásticas, individuales comunes y colectivas y otros tipos desarrollados por innumerables autores, sin embargo en este proyecto se parte principalmente de los postulados de Maslow, cuando se refiere a las necesidades fisiológicas, de seguridad, de pertenencia, de interacción social y de amor, de estima y autorrealización y especialmente resulta interesante desarrollar las denominas necesidades emocionales.

Cuando se hace referencia a una necesidad emocional, es necesario pensar en todo lo que resulta vital o indispensable para un individuo. En este sentido los bienes o servicios que el hombre utiliza para intentar satisfacer sus necesidades, le generan sensaciones y percepciones, que lo hacen sentirse a gusto o no con el instrumento utilizado y su entorno, donde por supuesto se encuentran las empresas que produjeron el bien o prestaron el servicio. Para entender este planteamiento con mayor profundidad es necesario presentar las teorías que permiten entender que es una emoción, sus elementos descriptivos, clases, importancia y en especial su relación con las necesidades, hasta desarrollar lo que conocemos como una necesidad emocional, las cuales varían en función de diferentes variables y condiciones propias de los seres humanos, tales como la edad.

Las emociones sirven para establecer nuestra posición con respecto a nuestro entorno, y nos impulsan hacia ciertas personas, objetos, acciones, ideas y nos alejan de otros. Las emociones actúan también como depósito de influencias innatas y aprendidas, y poseen ciertas características invariables y otras que muestran cierta variación entre individuos, grupos y culturas (Levenson, 1994). Según este importante autor, las emociones son determinantes de las relaciones interpersonales y con las organizaciones o redes, a las que incluso conforman las personas, por lo tanto son claves a la hora de satisfacer sus necesidades, dado que para hacerlo por naturaleza, al ser el hombre un ser social, necesita y recurre a otros, entre los que se encuentran las empresas que generan los bienes o prestan los servicios que se utilizan.

Es oportuno entonces argumentar teóricamente, lo que hemos llamado conexiones emocionales, en especial, dada su importancia tanto para los consumidores como para los productores o prestadores de un servicio, los cuales interactúan en los mercados y por lo tanto son los más interesados en sacar la máxima ventaja de esa relación, específicamente, desde la perspectiva del cliente, al obtener o recibir bines o servicios, con la mejor función costo beneficio, y desde la posición de la empresa, satisfacer las necesidades de sus demandantes, creando el mayor valor y margen posible, que se refleje en riqueza, sostenibilidad y sustentabilidad.

"El valor emocional es literalmente el valor económico de los sentimientos", Janelle Barlow (2000). Definitivamente las emociones están relacionadas con las necesidades y en especial con el deseo de alcanzar cierto producto o tener acceso a servicios, en este sentido cualquier empresa trabajara por activar, estimular y provocar dichas necesidades, hasta tal punto que logre conectarse emocionalmente con sus potenciales o habituales clientes. Por ejemplo, si un individuo tiene emociones positivas alrededor de sus deseos de ser Exitoso, importante, incluido, independiente o sencillamente notado, podrá identificarse y como tal sus emociones genérale impulsos que lo lleven a adquirir ciertos bienes o comprar servicios, a través de los cuales logre vivir una experiencia de satisfacción emocional. En este contexto la empresa venderá y como tal se sustenta el valor económico de las emociones.

Es importante aclarar que las emociones pueden ser positivas o negativas y en ambas condiciones, resultan para un consumidor y por su puesto para el ofertante de un bien o servicio, clave su identificación. Es este sentido la investigadora Bárbara Fredrickson ha abierto una línea de investigación (Fredrickson, 1998, 2000b, 2001, 2003; Fredrickson y Branigan, 2000). Recientemente ha planteado la Teoría abierta y construida de las emociones positivas (Broaden and build theory of positive emotions) (Fredrickson, 1998, 2001), 
que sostiene que emociones como la alegría, el entusiasmo, la satisfacción, el orgullo, la complacencia, etc., aunque fenomenológicamente son distintas entre sí, comparten la propiedad de ampliar los repertorios de pensamiento y de acción de las personas.

A partir de los conceptos teóricos planteados, se propone la siguiente definición de Conexión Emocional:

"Las conexiones emocionales representan el tejido neuronal que crea la sinergia entre los bienes o servicios, que producen o prestan las empresas con la intención de satisfacer las necesidades superiores del hombre, logrando que este perciba un cumplimiento de la promesa de valor que le fue realizada, mediado este proceso por la activación de emociones positivas que dan cuenta de una experiencia satisfactoria, que motiva a la compra creando relaciones comerciales y sociales duraderas, e inspirando al oferente a una mejora continua en el diseño y prestación del servicio"

\section{Metodología}

\section{La metodología para el desarrollo de esta investigación se planteó en las siguientes fases}

Fase 1: Se comenzó con una revisión bibliográfica acerca del estado del arte de las estrategias de servicio de los últimos 20 años.

Fase 2: Se identificaron algunas de las empresas de talla mundial que desarrollaran estrategias de servicios, para luego pasar a investigar acerca de las estrategias de servicio que han implementado. Los criterios de selección son los siguientes:

Encontrarse en el listado de las 500 mejores empresas de acuerdo a la selección que realiza la revista Forbes 500.

La empresa seleccionada debía tener dentro de su portafolio de productos un alto componente de servicios y a su vez que sean servicios que satisfacen necesidades de orden superior.

Sumando a los anteriores criterios, se tuvo en cuenta la labor propia de un investigador en relación que si lo que se quiere investigar es posible investigarlo, en términos de acceso a la información.

El número de empresas a seleccionar se dio en función del ejercicio de la investigación, en la medida que se pueda seguir la huella del tema elegido y que se le hace posible investigar con las herramientas que se tiene a nivel de investigación pues no todas las empresas han identificado las emociones como herramientas estratégicas para crear valor.

Fase 3: Con base en el estudio de los casos más representativos, se procedió a realizar un análisis cualitativo de los atributos más determinantes en el éxito de estas organizaciones, soportado en estudios de casos realizados por universidades reconocidas internacionalmente (Harvard, Incae, etc).

Fase 4: Se planteó la aproximación a un modelo teórico de estrategia no convencional de servicios que agrupe las variables más relevantes del análisis anterior, junto con la teoría escrita previamente analizada. Para verificar la validez de dicho modelo se plantea la comparación con otras empresas del mercado global.

Fase 5: Por último, se finalizó el proyecto revisando nuevamente la bibliografía, ajustando tanto el modelo como el marco teórico según los hallazgos encontrados para evidenciar las brechas existentes entre las estrategias de servicio seguidas por las organizaciones modernas y la bibliografía relativa a este tema.

\section{Resultados}

Luego de realizar un análisis de tres de las más importantes empresas del mundo que soportan un alto componente de su creación de valor en el servicio, según el listado de la revista "Forbes", previo estudio en profundidad del marco conceptual, teórico y referencial que sustentan las diferentes tesis ideológicas que han abordado el núcleo problémico de las emociones, desde la perspectiva empresarial y financiera, en el marco de los intereses por preservar la clientela y mejorar la posición en el mercado a partir de ventajas competitivas que permitan un aumento de los consumidores potenciales y reales, con 
criterios de sostenibilidad y sustentabilidad, se logró entonces, formular una hipótesis de trabajo fundamentada el estudio de las conexiones emocionales, como fuente de información, base para el diseño y desarrollo de servicios innovadores y que se califiquen dentro de los ámbitos de las estrategias no convencionales de servicio. Por lo tanto, a continuación se pretende realizar una abstracción teórica del mundo real de las empresas de talla mundial analizadas, con la firme intención de construir y presentar un modelo que reduzca la complejidad técnica del tema abordado y sus múltiples dimensiones. Así mismo que ayude a docentes, investigadores y científicos a realizar predicciones concretas que dirijan $u$ orienten los estudios empíricos que se inspiren en estos postulados.

La posición conceptual en la cual se cimienta la construcción de esta propuesta de modelo, parte de la convicción de este constituye un bosquejo que representa una realidad construida de variables y postulados ideológicos y que tiene como objetivo describir, explicar, analizar y comprender la realidad del fenómeno de las conexiones emocionales como elemento nuclear en las estrategias no convencionales de servicio, que para este caso se hace más útil en virtud de la enorme dificultad para estudiar la realidad de las emociones en sí mismas, desde la perspectiva física y en la intangibilidad de las conexiones emocionales que se tejen a nivel neuronal, producto de las sinergias creadas entre los servicios recibidos por los usuarios o clientes.

De acuerdo con el tipo de ciencia en la cual el investigador se ubique, se puede encontrar a nivel teórico diferentes clasificaciones de los modelos: Verbales, de simulación, analíticos, el numérico, el matemático, el físico entre otros, sin embargo, tomando en cuenta el construido y que se socializará en este capítulo, corresponde este a un modelo científico, que se caracteriza por su enfoque abstracto de la realidad, partiendo de un marco conceptual que se intenta sintetizar, liberándolo de encrucijadas y presentándolo en un lenguaje gráfico, visual de acuerdo con la dimensión cualitativa o cuantitativa que este tenga.

En relación a los tipos de modelos que se tomaron como base, se encuentran los de orden cualitati- vo, los cuales sirven o intentan determinar las relaciones entre diferentes variables o factores que integran un sistema. En este caso en particular, es importante recordar que se sustentó en el sistema que representa el proceso, por medio del cual las organizaciones formulan e implementan estrategias no convencionales de servicio, generadoras de una ventaja competitiva, fundamentadas en la relación entre necesidades superiores, los bienes o servicios, mediada esta relación por las emociones a partir de lo cual se generan conexiones emocionales positivas o negativas. Los modelos cualitativos no cuantifican dichas relaciones, solamente facilitan su entendimiento. Es decir, ayudaría a entender la sinergia entre las variables, la forma en la que estas se relacionan, por lo tanto es vital para comprender la conexión emocional, que precisamente da cuenta del proceso específico de interés.

La construcción de este tipo de modelos, exigen gran creatividad, y suelen ser de muy oportunos para explicar relaciones que parten desde planteamientos sencillos, pero que conforme se desarrollan, crecen en variables, dimensiones que se pueden involucrar con facilidad. Es esta cualidad, lo que los hace interesante y altamente útil.

Tomando en cuenta los elementos anteriormente expuestos a continuación se presentan dos modelos construidos a partir del análisis realizado a las empresas de talla mundial que han reconocido la importancia de las emociones como mediadoras de la relación existente entre las necesidades superiores y los bienes y servicios que producen o prestan estas empresas:

\section{Modelo de conexión emocional}

\section{Eje temático:}

Conexiones emocionales como fuente de ventaja competitiva en las estrategias de servicio.

\section{Contexto:}

Este modelo es el producto de un análisis de empresas de talla mundial en la búsqueda de codificar cómo ellas crean valor a través de estrategias no convencionales basadas en las conexiones emocionales con sus clientes a través del servicio. 


\section{Variables clave}

Conexión emocional, creación de valor, proceso emocional, proceso racional, razón, emoción, el triángulo de necesidades de Maslow, necesidades básicas y superiores, emociones (pasión, pertenencia, reconocimiento, confianza, aspiraciones), Fidelidad, devoción, mayores compras y referidos, ventajas comparativas y competitivas, estrategia convencional y estrategia no convencional.

\section{Tipo de modelo}

El tipo de modelo se ajusta a la categoría de un modelo científico de orden cualitativo, gráfico y visual, que pretende describir una abstracción teórica de una realidad de las empresas analizadas. Es puramente cualitativo en la medida que las emociones son un concepto puramente intangible contra las variables económicas que pueden ser plasmadas en resultados financieros.

\section{Objetivo}

Es un punto de partida para contextualizar e inspirar a otras investigaciones que traten de aproximarse a una conceptualización de estrategias basadas en variables de tipo emocional correlacionadas con variables socioeconómicas. Teniendo en cuenta que el $80 \%$ de las empresas de Cartagena pertenecen al sector de servicios y necesitan encontrar información sobre la cual construir nuevas estrategias para mejorar su posición en el mercado e incrementar la creación de valor, este proyecto se convierte también en un punto de partida con el propósito de inspirar a investigadores regionales para que puedan diseñar, construir e implementar modelos para contextualizar las estrategias no convencionales de servicio en los mercados locales o regionales de Colombia.

\section{Elementos fundamentales del análisis cualitativo}

\section{Descripción}

El modelo de conexión emocional parte de la relación estrecha que existe entre la creación de valor y la ventaja competitiva. La creación de valor se da cuando el cliente adquiere un servicio y posteriormente este se traduce en flujos de caja que permitan a una empresa mantenerse, crecer y lograr la sostenibilidad. Sin duda el cliente es en donde se origina la creación de valor, es por tanto indispensable entender por qué una persona decide por una compra, cuáles son sus motivos, sus expectativas, sus convicciones, lo que espera del servicio. El ser humano al ser un individuo biopsicosocial, tiene unas necesidades básicas y superiores que satisface cuando adquiere el servicio. Se ha encontrado que los servicios que una persona consume para la satisfacción de sus necesidades básicas crean en el individuo unos hábitos de consumo racionales que lo convierten en un comprador racional el cual está enmarcado por atributos como la fidelidad, devoción, referidos y la necesidad de compras de más servicio. Para las compañías, estos clientes le reportan ventajas comparativas, que son fáciles de imitar y son de poca duración.

Pero los individuos son seres dinámicos y evolucionan hacia estilos de vida diferentes; cuando una persona ya ha satisfecho sus necesidades básicas, apunta a cubrir sus necesidades superiores, esas necesidades van más de la mano a estatus de reconocimiento social y autorrealización, por lo tanto sus decisiones sobre el servicio a consumir ya no dependen de su razón, sino es complementada con sus emociones, siendo estas últimas las de mayor importancia que las de la razón, adquiere un servicio es para sentirse bien. El modelo lo que pretende explicar a las empresas es este comportamiento del cliente y sabiendo esto, los clientes de los servicios están en una dinámica de permanente monitoreo en donde hay que visualizar necesidades, procesos racionales, emociones y decisión de compra. La espiral en el modelo (ver fig. 3) representa el proceso de creación de la conexión emocional, la cual no se da de manera inmediata sino que parte de una serie de detalles (proceso racional) que provocan una reacción emocional en el individuo y esta a su vez se retroalimenta y se amplifica en la medida que la experiencia haya sido positiva y que la sucesión de eventos racionales sigan reafirmando ese proceso de amplificación de las emociones hacia la organización hasta llegar a crear un vínculo suficientemente sólido que despierte la pasión por la empresa o por la marca que representa un estado de logros superior. A partir de estas conjeturas se propone el siguiente modelo conceptual que describe en forma gráfica el fenómeno de vinculación de las emociones con la decisión de compra (ver fig. 3) 


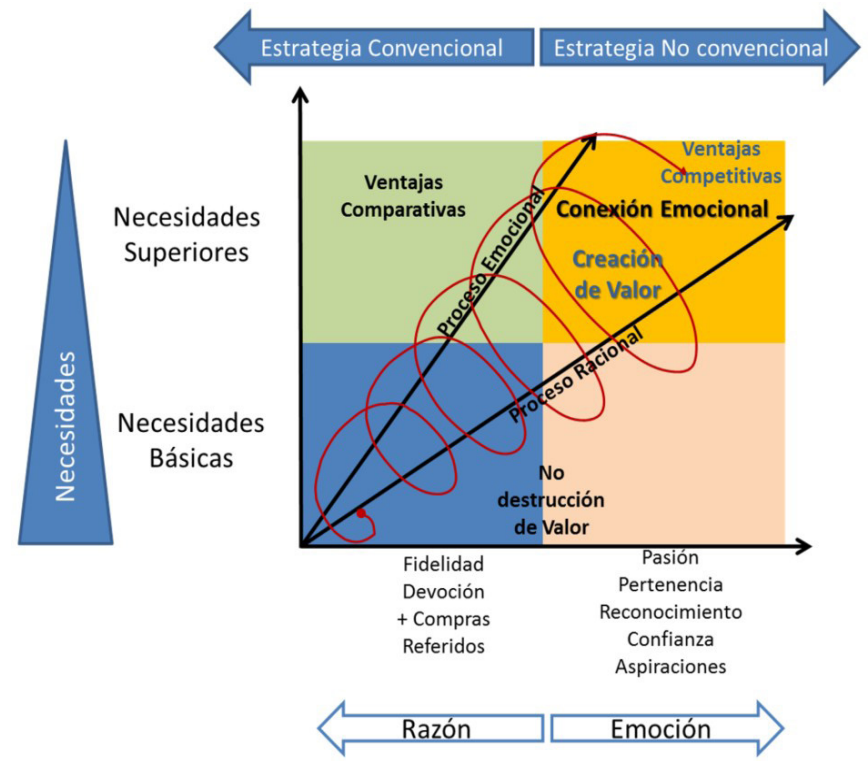

Figura 3. Modelo de conexión emocional.

Fuente: Elaboración propia

\section{Explicación}

Ese estado que genera las emociones de pasión, pertenencia, reconocimiento, confianza y aspiraciones se ha identificado como la base de las estrategias no convencionales en el servicio, dado que apuntan a satisfacer necesidades de orden superior. Si la empresa a través de la ejecución del servicio, logra establecer estos vínculos emocionales que permitan mediante la adquisición de dicho servicio satisfacer las necesidades superiores, logrará una ventaja competitiva en su mercado. Estos vínculos son los que hacen que un cliente adquiera el servicio de forma no racional sino emocional, motivada por la pasión, la aspiración, confianza, pertenencia o reconocimiento hacia el mismo.

\section{Análisis}

Cumplir o inclusive superar las expectativas del cliente no es suficiente, sino que se debe lograr esa conexión emocional que se describe en el modelo. Esta no se da de manera inmediata sino a través de las experiencias previas positivas en el servicio recibido, sumando también los detalles racionales que van creando un proceso emocional de manera repetitiva y cíclica tal como se muestra en la espiral de la fig. 3. Al repetirse estas experiencias positivas se va afianzando la conexión emocional, se entra en la generación de confianza del cliente hacia la organización y a medida que crece este proceso emocional se incrementa la fidelidad y devoción del cliente hacia la empresa lo cual se traducirá en compras repetidas y en referenciación hacia otros clientes potenciales. De esta forma se producirá la ventaja competitiva que indudablemente llevará a la creación sustentable de valor.

\section{Para qué es útil}

La utilidad de este modelo se fundamenta en el supuesto que, de la hipótesis planteada puede surgir un proyecto de investigación como base para aceptar o rechazar dicha hipótesis en un contexto de mercado local como el de la ciudad de Cartagena, para poder desarrollar futuras investigaciones de tipo cuantitativo.

Se puede clasificar la utilidad de la aplicación de este modelo en tres dimensiones del conocimiento aplicado: la dimensión económica, la social y la tecnológica.

Desde el punto de vista de la dimensión económica, la utilidad de este modelo es la más evidente puesto que el mundo globalizado de hoy exige a las organizaciones la constante búsqueda de herramientas que les permitan lograr una posición de ventaja competitiva en su sector. El hecho de que este modelo inspire a los empresarios a explorar este tema bajo la concepción de conexiones emocionales como estrategia no convencional de servicio con el fin de mejorar la experiencia del cliente y que esta se vea reflejada en la 
creación de valor y en las condiciones financieras de la organización, reafirma la importancia del modelo puesto que el fin último de las organizaciones es la creación de valor como fuente de sustentabilidad.

Desde la dimensión tecnológica la utilidad de esta propuesta de modelo es la de estar aportando la posibilidad de crear un nuevo conocimiento que se hizo a partir de otros postulados académicos desde el punto de vista de conexiones emocionales donde se reconocieron unas variables y se creó un nuevo postulado que deja abiertas muchas posibilidades de investigación sobre el tema en cuanto a la relación que hay entre las emociones y la creación de valor en las organizaciones.

Desde la dimensión de la utilidad social, el hecho que este modelo inspire a empresas y académicos a investigar las emociones y eso sirva como base para rediseñar los servicios que les permitan vivir experiencias satisfactorias, hace que quien más esté interesada es la sociedad misma, receptora de esos servicios porque van a satisfacer sus necesidades de orden superior, y se va a generar una posición no de resistencia sino de agrado, satisfacción, confianza, pertenencia y aspiraciones. De esta manera el modelo puede ser de utilidad para mejorar la calidad de vida de los miembros de la sociedad como clientes de las organizaciones donde este pueda ser aplicado.

\section{Modelo de ventaja competitiva a partir de la estrategia no convencional}

\section{Eje temático}

Conexiones emocionales como fuente de ventaja competitiva en las estrategias de servicio.

\section{Contexto}

Este modelo se construyó como complemento al anterior, teniendo en cuenta no solamente el proceso de creación de la conexión emocional sino con una visión holística de como la conexión emocional puede ser aprovechada por la organización, generando retroalimentación, aprendizaje y de esta manera innovando en el servicio para llegar a la creación de valor.

\section{Variables Clave}

Cliente, Necesidades básicas y superiores, diseño del servicio, servicio entregado, estrategia no convencional de servicio, recursos y capacidades, servicio percibido, feedback, aprendizaje, fidelidad, devoción, +compras, referidos, innovación, creación de valor, ventaja competitiva.

\section{Tipo de modelo}

Al igual que el modelo anterior, el tipo de modelo se ajusta a la categoría de un modelo científico de orden cualitativo, gráfico y visual, que pretende describir una abstracción teórica de una realidad de las empresas analizadas. Es puramente cualitativo en la medida que las emociones son un concepto puramente intangible contra las variables económicas que pueden ser plasmadas en resultados financieros.

\section{Objetivo}

Este modelo se propone como complemento al modelo de conexión emocional, resaltando la importancia que tiene la retroalimentación hacia la estrategia de la organización en la medida que se vaya logrando la conexión emocional y se puedan identificar cuáles son los elementos que la constituyen con el fin de, a través del proceso de aprendizaje organizacional, llegar a realizar los ajustes estratégicos necesarios con el fin que el resultado se amplifique y permita llegar a la creación de valor que de origen a la ventaja competitiva.

\section{Elementos fundamentales del análisis cualitativo}

\section{Descripción}

A continuación se presenta el modelo de ventaja competitiva a partir de la estrategia no convencional, planteado desde la perspectiva de aprendizaje organizacional, en el cual la estrategia debe nacer a partir de la detección de las necesidades del cliente, teniendo en cuenta los recursos y capacidades de la organización. A partir de estos se diseña el servicio en todos sus detalles el cual va a ser presentado al cliente en forma de proceso, atributos, detalles, libretos, etc. El cliente al recibir el servicio tendrá una percepción de valor que es donde se genera el proceso emocional (positivo o negativo). En el caso que esta reacción emocional sea positiva, esta conllevará a la fidelidad, devoción y más compras, además de los referidos. Siendo así se genera un feedback o retroalimentación hacia la empresa que, de ser adecuadamente gestionada a través de pro- 
ceso de gestión del conocimiento, se da cabida al aprendizaje necesario para capitalizar esto en una innovación la cual hacia el cliente afianzará su conexión emocional a un nivel superior de reconocimiento, pasión, confianza, aspiraciones y pertenencia, mientras que hacia la organización esto redundará en creación de valor y ventaja competitiva, tal como se expresa en el segundo modelo planteado en la fig. 4.

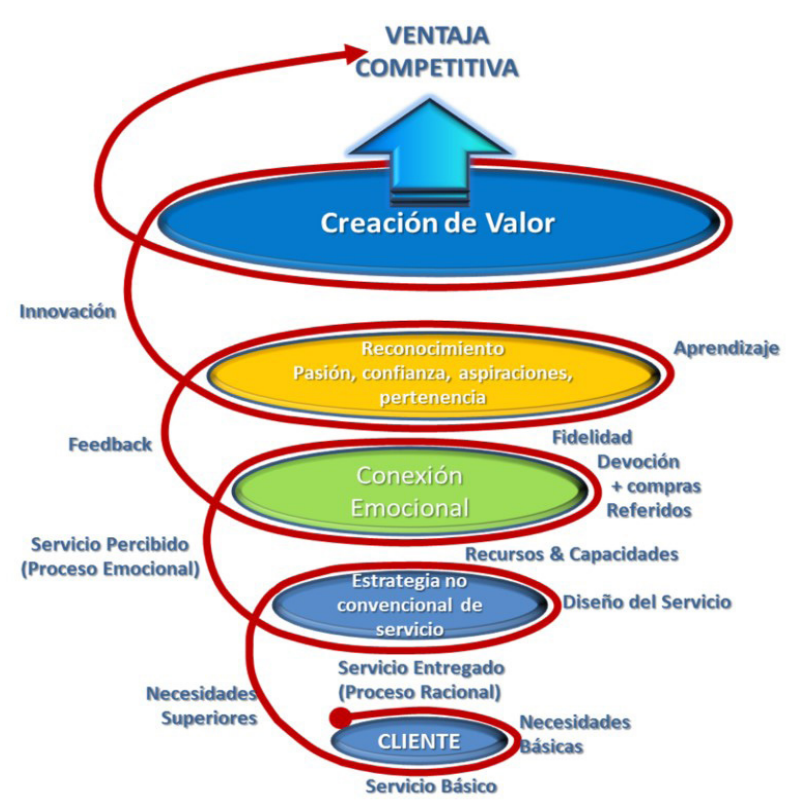

Figura 4. Modelo de Ventaja Competitiva a partir de estrategia no convencional.

Fuente: Elaboración propia

\section{Explicación}

Es importante anotar que debe haber una visión holística para entender que la conexión emocional abarca a todas las dimensiones de la organización y la relación con su entorno, haciendo de esto un proceso dinámico, que se retroalimenta y a la vez se amplifica a través del aprendizaje, gestión del conocimiento e innovación (Robledo y Del Rio, 2012) para llegar finalmente a la creación de valor sustentable que lleve a la organización a obtener su ventaja competitiva.

\section{Análisis}

Tanto este modelo como el anterior fueron inspirados en el análisis en profundidad de las empresas de talla mundial seleccionadas, tomando como referencia su experiencia positiva en cuanto a la construcción e implementación de estrategias no convencionales de servicio, que han generado objetivos intermedios, tales como la ventaja competitiva, por medio de la cual han logrado crear valor y riqueza, reflejada en el margen producto de las operaciones y viceversa. En ese orden de ideas resulta pertinente traer a colación los principales elementos que han hecho de estas empresas proveedoras no solo de bienes que satisfacen necesidades, sino que adicionalmente quienes los consumen logran vivir una experiencia satisfactoria. Por lo anterior, se procede de manera inmediata a socializar las principales conclusiones construidas, en virtud de la situación problemática de donde se partió y en especial los objetivos que fueron la ruta de inspiración, por lo tanto se pretende dar cuenta de los aspectos positivos y negativos directamente relacionados con estos.

\section{Para qué es útil}

Al ser este modelo un complemento al anterior, la utilidad de ambos cobra dimensiones similares, por lo tanto se hace referencia a la misma utilidad. Es de resaltar que el modelo puede servir de referencia para las empresas nacionales que quieran observar la creación de valor a través de la conexión emocional de sus servicios con sus clientes y desde esta perspectiva encontrar nuevas oportunidades para ajustar sus estrategias y así encaminarse a una nueva dimensión en el servicio que les permita incrementar su creación de valor. 
Estas estrategias y modelos son de particular interés hacia el sector público en algunas regiones de Colombia, las cuales adolecen de una cultura ciudadana que tenga una conexión emocional no solo con su ciudad sino con sus instituciones, generando una cultura de desprecio e irrespeto por su propio lugar donde habitan. Su aplicabilidad va desde instituciones de educación como colegios y universidades, entidades de salud, hasta entidades de gobierno como alcaldías, gobernaciones, organismos descentralizados, organizaciones militares, etc.

\section{Conclusiones y discusión}

La humanidad crece a un ritmo positivo, sin embargo los recursos disponibles para satisfacer sus necesidades, son cada vez más limitadas y escazas, dificultando su capacidad para tener acceso a bienes y servicios que generan las empresas que ellos crean, conforman y gestionan. Estas condiciones y situaciones les significan a muchos individuos problemas económicos a la hora de intentar satisfacer sus necesidades, y a las organizaciones serias dificultades para mantener sus clientes y su posición en el mercado, pero mucho más difícil es cautivar nuevos consumidores. A esta situación hay que sumarle las nuevas tendencias y dinámicas de los mercados, en donde hoy no solo se tienen competidores locales, dado la apertura económica de los países y la globalización, la cual trae consigo mayor cantidad de rivales, por lo tanto más alternativas, favoreciendo a los usuarios o demandantes, quienes ante esta condición de mercado premian el liderazgo en precio y el servicio.

Es precisamente el contexto descrito en medio del cual surgió la idea de investigación que se constituyó en una verdadera fuente de motivación, para emprender una búsqueda e indagación, sobre la cual abordar una de las preocupaciones más importantes de las empresas de hoy, ¿Cómo lograr mantener la posición en el mercado que poseen?, ¿Cómo mejorar la posición en el mercado?, ¿Cómo crear valor?, ¿Cómo crear riqueza con criterios de sostenibilidad?, ¿cómo enfrentar a los rivales y competidores?, muchas de estas preguntas contribuyeron a formular una inquietud que despertó el interés por lo innovador del mundo de los negocios y especialmente en las empresas de servicio que están preocupadas por optimizar su condición y resultados financieros, para lo cual comprenden que es necesario formular, ejecutar e implementar estrategias, que más allá de lo convencional contribuyan a la creación de ventajas competitivas sostenibles, que conduzcan a la organización hacia sus objetivos. Fue así como se formuló la pregunta de investigación: ¿Cuáles son los elementos que caracterizan las estrategias no convencionales de servicio que se utilizan en empresas de talla mundial?

Con la intención de dar respuesta a este núcleo problémico, se estableció como objetivo caracterizar las estrategias no convencionales de servicio aplicadas en el diseño, ejecución y creación de valor en empresas de talla global. Considerando que al alcanzar este resultado se contaría con suficientes elementos para dar respuesta a la pregunta de investigación. Al respecto, inicialmente en este proyecto se realizó un estado del arte a través del cual se construyó un marco teórico conceptual y referencial, analizando en profundidad las variables o categorías fundamentales tales como: las necesidades superiores, las emociones, la conexión emocional y las estrategias no convencionales.

Al comienzo de la investigación, se tenía un enfoque más operativo del servicio, sin embargo al revisar el estado del arte se fue descubriendo el protagonismo y la gran importancia que cobra la conexión emocional en el servicio lo cual provocó un giro importante en la investigación y llevó a sus autores a contemplar nuevos aspectos no conocidos previamente, generando un gran interés por continuar y plantear investigaciones futuras relacionadas con el tema.

Desde el punto de vista del análisis realizado a las empresas, se constata que todas ellas comparten unos elementos comunes relacionados con la conexión emocional, independiente de sus actividades económicas y la apuesta por esta estrategia les ha significado el aumento consistente en la creación de valor.

Una vez realizado el estado del arte que se planteó en el presente trabajo, se logró caracterizar las estrategias no convencionales de servicio en aspectos como la conexión emocional, la relación 
cíclica entre los procesos racionales y emocionales, los aspectos psicológicos de las interacciones humanas, la importancia de las relaciones del empleador con el personal de contacto. Estas conexiones emocionales están íntimamente ligadas a suplir las necesidades de orden superior de los clientes. Las decisiones de compra de los clientes están vinculadas de una manera más cercana a las emociones que a lo racional, cuando se trata de suplir necesidades de orden superior.

En línea con los objetivos, se logró construir un modelo que precisamente tiene la intención de constituirse como una aproximación conceptual al proceso de generación de estrategias no convencionales de servicio, que describe gráficamente sus elementos constitutivos y su impacto en la creación de valor, destacándose la conexión emocional como protagonista, y los procesos racionales y emocionales como vehículo para llegar a construir dicha conexión. De igual forma se planteó un segundo modelo complementario teniendo en cuenta la retroalimentación y la amplificación que se logra a través de esta conexión emocional cuando se gestiona a través del aprendizaje organizacional.

Adicionalmente, se construyó una hipótesis de trabajo que se deja planteada como punto de partida para futuras investigaciones que darán cuenta de su validez y relevancia.

Desde el punto de vista académico y particularmente en el campo de la administración, se invita a los investigadores interesados en este tema de trascendental importancia para la industria nacional, ampliar la presente investigación hacia dimensiones de tipo cuantitativo, con el fin de definir la métrica de las conexiones emocionales y cuantificar la correlación de la creación de valor respecto a las conexiones emocionales y de esta manera suplir la falta de conocimiento que existe en Colombia alrededor de estos tópicos.

Cualquier investigación que tenga que ver con la creación de valor, la sustentabilidad y las ventajas competitivas deben ser vistas a través de las conexiones emocionales como punto crítico en la relación que existe entre los productos y servicios con la creación de valor.

Finalmente es importante concluir que los gerentes de hoy, son ejecutivos que deben tener el mundo en sus mentes. Deben igualmente ser planificadores, proactivos y con gran capacidad para asumir la responsabilidad general de gestionar los recursos, los ingresos y costos de la empresa, para tal fin lo más probable es que en el marco de sus responsabilidades de mercadeo, ventas y servicios, a nivel estratégico, pueda entonces encontrar en las conexiones emocionales, el contexto ideal sobre el cual fundamentar el diseño e implementación de estrategias y servicios no convencionales, creadores de valor, de ventajas competitivas y posición optima en el mercado con resultados financieros adecuados y sostenibles, a partir de las relaciones comerciales más fuertes y duraderas que le significan a tanto el consumidor como el productor o prestador del servicio, vivir experiencias significativamente satisfactorias.

\section{Referencias bibliográficas}

A.Parasuraman, V. A. (1985). A Conceptual Model of Service Quality and Its Implications for future Research. Journal of Marketing, Vol 49, No.4, 41-50.

Albrecht, K., \& Zemke, R. (2000). Gerencia del Servicio: Cómo hacer negocios en la Nueva Economía. Bogotá: 3R Editores.

Alcaide, J. C. (22 de 02 de 2008). El caso Walt Disney: ¿¿un modelo de calidad, servicio y experiencia?? Recuperado el 06 de 09 de 2013, de MDS Servicio, Fidelización, Marketing y Ventas: http://www.marketingdeservicios.com/blog/fidelizacion-de-clientes/el-caso-walt-disney- $\% \mathrm{C} 2 \% \mathrm{BF} \% \mathrm{C} 2 \% \mathrm{BFun}-$ modelo-de-calidad-servicio-y-experiencia/

Al-Dweeri, R. M. (2011). La Calidad en los Servicios Electrónicos como Estrategia Competitiva. Modelo de Análisis de sus Componentes y Efectos sobre la Satisfacción y la Lealtad (Tesis Doctoral). Málaga: Servicio de Publicaciones de la Universidad de Málaga. 
Francisco Vergara Streinesberger, Juan Carlos Robledo Fernandez, Zully Aguilera Prins, Gustavo

Camargo Gutierrez

Apple inc. (2013). Recuperado el 10 de 09 de 2013, de http://www.apple.com/apple-events/september-2013/

Barlow, J., Maul, D., \& Edwarson, M. (2000). Valor Emocional: Creando lazos fuertes con sus clientes. San Francisco: Berrett-Koehler.

Boeree, G. (2006). Abraham Maslow. Recuperado el 07 de 09 de 2013, de Personality Theories: http://webspace.ship.edu/cgboer/maslow.html

Bolton, R., \& Drew, J. (1991). A Longitudinal Analysis of the impact of service changes on customer attitudes. Journal of Marketing 55, 1-9.

Bonchek, M. (2012). Putting Facebook in Perspective. Boston, Massachusetts, United States: Harvard Business Review Blog.

Braidot, N. (2006). Neuromarketing: Neuroeconomía y Negocios. Nestor Braidot.

Charam, R. \&. (2009). Cambio de Juego. Bogotá: Norma.

Chóliz, M. (2012). Psicología de la Motivación y Emoción. Recuperado el 07 de 09 de 2013, de Página de Mariano Chóliz Montañés: http://www.uv.es/=choliz/indicemotivacion2011.htm

Cooper, R., \& Sawaf, A. (1998). La Inteligencia Emocional Aplicada al Liderazgo y a las Organizaciones. Bogotá: Norma.

Damasio, A. (1999). El Error de Descartes: La Razón de las Emociones. Santiago de Chile: Andrés Bello.

De Ríos, C. C. (2018). ¿Cuál es la importancia de implementar estrategias en las organizaciones? Revista científica anfibios, 1(1), 71-79.

Díaz, L. (29 de 05 de 2011). Google se reinventa: reduce el tiempo de búsqueda y crea su propia computadora. Recuperado el 12 de 09 de 2013, de Perfil.com: http://www.perfil.com/ediciones/ ciencia/-20115-576-0065.html

Edelman, B., \& Eisenman, T. (2010). Google Inc. Caso 910-S15. Boston: Harvard Business School.

Elster, J. (2002). Alquimias de la mente: La racionalidad y las emociones.

Fleming, J., Coffman, C., \& Harter, J. (1995). Manage Your Human Sigma. Harvard Business Review, 107-114.

Fortune. (2019). Fortune Global 500 List 2018: See Who Made It.

Fredrickson, B., Tugade, M., Waugh, C., \& Larkin, G. (2003). What Good are positive emotions in crises? A prospective study of resilience and emotions following the terrorist attacks on the United States of September 11th, 2001. Journal of Personality and Social Psychology, 84, 365376.

Frei, F. \&. (2012). Uncommon Service. Boston Massachusetts: Harvard Business Review Press.

Gans, J. (2012). Disney Nailed Attention to Detail Long Before Apple.Recuperado el 09 de 09 de 2013, de Harvard Business Review Blog Network: http://blogs.hbr.org/cs/2012/03/the_mouse_on_the_manhole.html

García, O. (2009). Administración Financiera, Fundamentos y Aplicaciones, 4 ed. Cali: Prensa Moderna. 
Garrido Moreno, A. (2008). La Gestión de Relaciones con Clientes (CRM) como Estrategia de Negocio: Desarrollo de un Modelo de Éxito y Análisis Empírico en el Sector Hotelero Español (tesis doctoral). Málaga: Servicio de Publicaciones de la Universidad de Málaga.

Gary, L. (2004). The Science - and poetry- of Knowing Customers. Harvard Managemente Update. Google Inc. (2013). Acerca de Google.

Hallowell, R. (1996). The relationships of customer satisfaction, customer loyalty and profitability: an empirical study. International Journal of Service Industry Management, Vol. 7 No. 4, 27-42.

Herzberg, F., Mausner, B., \& Bloch Snyderman, B. (2009). The Motivation to Work. Washington: Library of Congress.

Interbrand. (2012). Best Global Brands 2012 Rankings

Jojn R. Tauser and Kenneth J, W. (1982). Dynamic Analysis of Consumer Response to marketing strategies. management science Vol 28, No 5, 455-486.

Jones, B. (2013). 3 FORMS OF GENUINE CARE THAT MAKE ALL THE DIFFERENCE.

Kotler, P., Cámara, D., Grande, I., \& Cruz, I. (2000). Dirección de Marketing. Madrid: Pretince Hall.

Levenson, R. (1994). Human Emotion: A Functional View. Oxford: PG Davinson, RJ.

Lopez, B. (2006). Publicidad Emocional, Estrategias Creativas. Madrid: ESIC .

Lovelock, C., Reynoso, J., D’Andrea, G., \& Huete, L. (2004). Administración de Servicios. México: Pearson Educación.

Lusch, R. \&. (2006). The Service-dominant Logic of Marketing: Dialog, Debate, and Directions. New York: M.E. Sharpe, Inc.

Ma, C., Wang, Z., Xu, X., \& Wu, Q. (2013). Measuring Service Value Based on Service Semantics. Journal of Service Science and Management, 56-68.

Marín Ximenez, N., \& Sanz, L. (Julio de 2013). Apple Incorporated. INCAE Business School. Alajuela, Costa Rica.

Martinez Coll, J. (2001). Las necesidades sociales y la pirámide de Maslow. Recuperado el 05 de 09 de 2013, de eumed.net: http://www.eumed.net/cursecon/2/necesidades_sociales.htm

Maslow, A. H. (1991). Motivación y Personalidad. Madrid: Diaz de Santos S.A.

Porter, M. (1996). What Is Strategy? Harvard Business Review, 61-78.

Porter, M. (1998). Competitive Strategy: Techniques for Analyzing industries and competitors. Glencoe, Il: Free Press.

Reeve, J. (2009). Understanding Motivation and Emotion. Washington: Library of Congress.

Robledo, J. (2010) "La Mercadotecnia en las organizaciones: La Gestion del conocimiento en la Mercadotecnia”, Pp. 19-22, Ediciones ILCSA S.A, Primera edición. Universidad Autónoma de Baja California. ISBN 9786077736301 
Robledo, J., Del Rio, J., Ruiz, G., Martinez, O.(2015) “Gestion del Conocimiento Organizacional, Fundamentos Teóricos”. Jorale editores y Universidad Autonoma de Baja California (Mexico). ISBN 9786077522249.

Sarmiento, L. M. D. (2018). Gestión estratégica de proveedores para fortalecer el proceso de compras en la línea de negocios de reparación y mantenimiento de embarcaciones en COTECMAR. Revista científica anfibios, 1(2), 44-52.

Scott, R., Brand, C., \& Lenz, V. (2001). Márketing Emocional. Barcelona: Gestión 2000.

Sinek, S. (2009). Start With Why. New York: Penguin Books.

Slywotzky, A., \& Weber, K. (2012). Demanda: Crear lo que las personas desean antes de que sepan qué quieren. Bogotá: Buena Semilla.

The Walt Disney Company. (2013). Recuperado el 9 de 9 de 2013, de http://thewaltdisneycompany. com/about-disney/company-overview

Thomson, K., \& Tarodo, A. (2000). El Capital Emocional: Cómo cautivar los corazones y las mentes para conseguir empresas que triunfen a través del marketing y la comunicación interna. ESIC. 